\title{
Probléme de Somigliana sur la théorie de l'élasticité a modules différents dans un milieu orthogonal-anisotrope.
}

\author{
I. T. Mintchev (Sofia) (*)
}

\begin{abstract}
Resnmé. - On obtient les équations fondamentales du problème de Somigliana pour la thérie d'élasticité dans un milieu orthogonal anisotrope ò modules différents par l'intermédiaire des fonctions des contraintes $F(x, y)$ et $\psi(x, y)$.

Pour $F$ et on obtient un système d'équations differentielles partielles.

Il est montré, que si les points sont disposés sur la ligne isoclinne, la forme des fonctions $F^{\prime}$ et $\psi$ se determine exactement.

Sont étudiés aussi les cas oì le corps se trouve dans l'état de contrainte et de déformation planéaire.
\end{abstract}

\section{Introd action.}

Le problème posé par C. Somigliana exige que soient determinés les contraintes et l'état de déformation d'un corps elastique, limité par une surface cilyndrique, sous l'action d'une part de forces extérienres distribuées sur cette surface, d'autre part de forces volumiques. Les dimensions de la section transversale et la longueur du corps peuvenet être aussi bien limitèes qu'illimitées [1].

La résolution da problème posé, pour une anisotrope totale et sous condition que les forces volumiques sont sans potenciel, est donné par W. VoIGT [2]; mais quand les mêmes forces ont un potenciel, la solution est donnée par S. G. LECHNITZKI [3], [4].

Notre but est de resoudre le problème de Somighrara dans un milieu orthogonal-anisotrope à modules différents quand il y a de petites déformations.

$\S$ 1. Rapportons la corps à étudier un système de coordonées orthogona. les. L'axe $z$ est identique à l'axe du corps et $x, y$ sont identiques aveo les directions principales de l'orthotropie.

Comme il est montré dans notre travail [5], la loi genéralisée de Hooke pour le corps étudié a la forme.

$$
\begin{aligned}
& \varepsilon_{x x}=A_{11} \sigma_{x x}+A_{21} \sigma_{y y}+A_{31} \sigma_{z z}+A_{41} \sigma_{y z}+A_{51} \sigma_{x z}+A_{61} \sigma_{x y} \\
& \ldots \ldots \ldots \ldots \\
& \varepsilon_{x y}=A_{16} \sigma_{x x}+A_{26} \sigma_{y y}+A_{36} \sigma_{z z}+A_{46} \sigma_{y z}+A_{56} \sigma_{x z}+A_{66} \sigma_{x y}
\end{aligned}
$$

(*) Entrata in Redazione il 5 novembre 1968 . 
I. T. Mintchev: Probléme de Somigliana sur la théorie de l'élasticité, etc.

où $\varepsilon_{x x} \ldots$, et $\sigma_{x x}$, sont composantes du tenseur des déformations et des contraintes, $A_{i j}$ sont les coefficients de déformation qui sont fonction des cosinus directeurs des directions principales de l'orthotropie par rapport aux directions principales des contraintes.

Les coefficients prennent des valeurs differentes en fonction des signes des contraintes principales.

En général $A_{i j} \neq A_{j i}$.

De part la forme de la charge extérieure il suit que la partie droite de (1) est une fonction continue de $x$ et de $y$.

En ayant en vue les formules de Cauchy:

$$
\begin{aligned}
& \varepsilon_{x x}=u_{, x}, \quad \varepsilon_{y y}=v_{, y}, \quad \varepsilon_{z x}=w_{, z}, \\
& \varepsilon_{y z}=v_{, z}+w_{, y}, \quad \varepsilon_{x z}=u_{, z}+w_{, x}, \quad \varepsilon_{x y}=u_{, y}+v_{. x},
\end{aligned}
$$

après intégration de $z$ on determine les composantes du deplacement élastique dans un point quelconque quand les composantes des contraintes ne depen. dent pas de z.

De cette façon $u, v, w$ on trouve les expressions suivantes:

$$
\begin{aligned}
& u=-\left(A_{3 l} \sigma_{i j}\right)_{, x} \frac{z^{2}}{2}+\left(A_{5 l} \sigma_{i j}\right) z-w_{0, x} z+u_{0}, \\
& v=-\left(A_{3 l} \sigma_{i j}\right)_{, y} \frac{z^{2}}{2}+\left(A_{4 l} \sigma_{i j}\right) z-w_{0, y} z+v_{0}, \\
& \quad w=\left(A_{3 l} \sigma_{i j}\right) z+w_{0} ; \\
& (l=1,2, \ldots, 6 ; i, j=x, y, z) \\
& \left(A_{3 l} \sigma_{i j}=A_{31} \sigma_{x x}+A_{32} \sigma_{y y}+A_{33} \sigma_{z z}+A_{34} \sigma_{y z}+A_{35} \sigma_{x z}+A_{36} \sigma_{x y}\right) .
\end{aligned}
$$

Les expressions sont identiques aveo les mêmes expressions du deplace. ment élastiques de la théorie classique sur le corps anisotrope, mais les coefficients $A_{k l}$ sont différents. Les fonctions $u_{0}, v_{0}, w_{0}$ se determinent par les trois equations restantes de la loi de Hooke.

\& 2. - Comme on la sait par [4], les composantes des contraintes se determinent, dans les problèmes de l'espace, par deux fonctions de contraintes par l'intermédiaire:

(4) $\sigma_{x x}=F_{, y y}+\bar{U}, \quad \sigma_{y y}=F, x x+\bar{U}, \quad \sigma_{x y}=-F_{, x y}, \quad \sigma_{x z}=\psi_{, y}, \quad \sigma_{y z}=-\psi_{, x}$,

où $\bar{U}(x, y)$ est le potenciel elastique.

Quand on remplace (4) dans la relation de $S^{\text {t }}$ Venant on obtient un 
système d'équations différentes partielles pour la détermination des fonctions $F(x, y)$ et $\psi(x, y)$ :

$$
\begin{aligned}
& \beta_{22} F_{, x x x x}+\beta_{11} F_{, y y y y}-\left(\beta_{26}+\beta_{62}\right) F_{, x x x y}+\left(\beta_{12}+\beta_{21}+\beta_{66}\right) F_{x x y y}- \\
& -\left(\beta_{16}+\beta_{61}\right) F_{, x y y y}+\left(2 \beta_{22, x}-\beta_{62, y}\right) F_{, x x}+\left(2 \beta_{11 . y}-\beta_{61, x}\right) F_{, y y}+ \\
& +\left(2 \beta_{12, y}-2 \beta_{26, x} \quad \beta_{62, x}+\beta_{66, y}\right) F_{, x x y}+\left(2 \beta_{21, x}-2 \beta_{16, y}-\beta_{61 . y}+\beta_{66, x}\right) F_{, x y y}+ \\
& +\left(\beta_{12, y y}-\beta_{22, x x}-\beta_{62, x y}\right) F, x x+\left(\beta_{11, y y}+\beta_{21, x x}-\beta_{61, x y}\right) F, y y- \\
& -\left(\beta_{16, y y}+\beta_{26, x x}-\beta_{66, x y}\right) F_{, x y}-\beta_{24} \psi_{, x x x}+\beta_{15} \psi_{, y y y}+\left(\beta_{25}+\beta_{64}\right) \psi_{x x y}- \\
& -\left(\beta_{14}+\beta_{65}\right) \psi_{, x y y}-\left(2 \beta_{24, x}-\beta_{64, y}\right) \psi_{, x x}+\left(2 \beta_{15, y}+\beta_{65, x}\right) \psi_{, y y}+ \\
& +\left(2 \beta_{25, x}-2 \beta_{14, y}+\beta_{64, x}-\beta_{65, y}\right) \psi_{, x y}-\left(\beta_{14, y y}+\beta_{24, x x}-\beta_{64, x y}\right) \psi_{, x}+ \\
& +\left(\beta_{15, y y}+\beta_{25, x x}-\beta_{65, x y}\right) \psi, y=-\left(\beta_{21}+\beta_{22}\right) \bar{U}_{, x x}-\left(\beta_{11}+\beta_{12}\right) \bar{U}_{, y y}+ \\
& +\left(\beta_{61}+\beta_{62}\right) \bar{U}_{, x y}+\left(\beta_{61, y}+\beta_{62, y}-2 \beta_{21, x}-2 \beta_{22, x}\right) \bar{U}_{, x}+\left(\beta_{61, x}+\right. \\
& \left.+\beta_{62, x}-2 \beta_{11, y}-2 \beta_{12, y}\right) \vec{U}_{, y}+\left(\beta_{61, x y}+\beta_{62, x y}-\beta_{11, y y}-\beta_{12, y y}-\beta_{21, x x}-\right. \\
& \left.-\beta_{22, x x}\right) \bar{U}+\left[\frac{A_{36}}{A_{33}}(A x+B y+C)\right]_{, x y}-\left[\frac{A_{23}}{A_{33}}(A x+B y+C)\right]_{, x x}- \\
& -\left[\frac{A_{13}}{A_{33}}(A x+B y+C)\right]_{, y y} \text {; }
\end{aligned}
$$

(5)

$$
\begin{aligned}
\beta_{42} F_{, x x x}-\beta_{51} F_{, y y y}^{i}-\left(\beta_{52}-\beta_{46}\right) F_{, x x y}+\left(\beta_{56}+\beta_{41}\right) F_{, x y y}-\left(\beta_{52, y}-\right. \\
\left.\quad-\beta_{42, x}\right) F_{, x x}-\left(\beta_{51, y}-\beta_{41, x}\right) F_{, y y}+\left(\beta_{56, y}+\beta_{46, x}\right) F_{, x y}-\beta_{44} \psi_{, x x}- \\
-\beta_{55} \psi_{, y y}+\left(\beta_{54}+\beta_{45}\right)_{, x y}+\left(\beta_{54, y}-\beta_{44, x}\right) \psi_{, x}-\left(\beta_{55, y}-\beta_{45, x}\right) \psi_{, y}= \\
=\left(\beta_{41}-\beta_{42}\right) \bar{U}_{, x}+\left(\beta_{51}+\beta_{52}\right) \bar{U}_{, y}-\left(\beta_{41, x}-\beta_{42, x}-\beta_{51, y}-\beta_{52, y}\right) \bar{U}- \\
-\left[\frac{A_{43}}{A_{33}}(A x+B y+C)\right]_{, x}+\left[\frac{A_{53}}{A_{33}}(A x+B y+C)\right]_{, y}+2 v ;
\end{aligned}
$$

où $A, B$ et $C$ sont des constantes d'intégration qui caractèrisent la flexion du corps dans les plans $(X O Z)$. (YOZ). et la déformation dans le sens de l'axe $z$. $v$ est l'angle relatif de la torsion autour de l'axe $z . \beta_{i j}$ sont déterminés par $A_{i j}$ par l'intermédiaire de la formule

$$
\begin{aligned}
& \beta_{i j}=A_{i j}-\frac{A_{i 3} A_{j 3}}{A_{33}} \\
& (i, j=1,2, \ldots, 6) .
\end{aligned}
$$


Des fonctions $F$ et $\psi$ doivent satisfaire non seulement le système (5) mais aussi, la condition du contour de la section transversale, qui d'après la pro. blème de Somigliana, est donné par une force axiale $P_{z}$ et un moment avec ses trois composantes $M_{x}, M_{y}, M_{z}$.

La résolution générale da sistẻme pour les conditions ci-dessus est très difficile parce que les coefficients $\beta_{i j}$ sont variables.

Si les points où l'on cherche les contraintes et les deformations du corps sont situés sur le lignes isoclinnes de la section transversale, dono les coefficients $\beta_{i j}$ ont constants et le système (5) se simplifie. Dans ce cas quand on introduit les quatre différents opératenrs, le système (5) peut s'écrire symboliquement comme ceci :

$$
\begin{aligned}
& \left(L_{2} L_{4}-L_{3} L_{3}^{*}\right) F=f_{1}(x, y) L_{2}-f_{2}(x, y) L_{3}, \\
& \left.\left(L_{3} L_{3}^{*}-L_{2} L_{4}\right) \psi=f_{1}(x, y) L_{3}^{*}-f_{2}{ }^{\prime} x, y\right) L_{4}
\end{aligned}
$$

où differement $d u$ cas classique, ici les opérateurs differenciels ont la forme:

$$
\begin{aligned}
L_{2} & =\beta_{44} \frac{\partial^{2}}{\partial x^{2}}-\left(\beta_{45}+\beta_{54}\right) \frac{\partial^{2}}{\partial x \partial y}+\beta_{55} \frac{\partial^{2}}{\partial y^{2}}, \\
L_{3}^{*} & =-\beta_{42} \frac{\partial^{3}}{\partial x^{3}}+\left(\beta_{52}+\beta_{46}\right) \frac{\partial^{3}}{\partial x^{3} \partial y}-\left(\beta_{41}+\beta_{56}\right) \frac{\partial^{3}}{\partial x \partial y}+\beta_{51} \frac{\partial^{3}}{\partial y^{3}}, \\
L_{3} & =-\beta_{24} \frac{\partial^{3}}{\partial x^{3}}+\left(\beta_{25}+\beta_{64}\right) \frac{\partial^{3}}{\partial x^{3} \partial y}-\left(\beta_{14}+\beta_{65}\right) \frac{\partial^{3}}{\partial x \partial y^{2}}+\beta_{15} \frac{\partial^{3}}{\partial y^{3}} \\
L_{4} & =\beta_{22} \frac{\partial^{4}}{\partial x^{4}}-\left(\beta_{26}+\beta_{62}\right) \frac{\partial^{1}}{\partial x^{3} \partial y}+\left(\beta_{12}+\beta_{21}+\beta_{66}\right) \frac{\partial^{4}}{\partial x^{2} \partial y^{2}}- \\
& -\left(\beta_{16}+\beta_{61}\right) \frac{\partial^{4}}{\partial x \partial y^{3}}+\beta_{11} \frac{\partial^{4}}{\partial y^{4}},
\end{aligned}
$$

où

$$
\begin{aligned}
& \beta_{i j}+\beta_{j i}, \\
& f_{1}(x, y)=-\left(\beta_{21}+\beta_{22}\right) \bar{U}_{, x x}-\left(\beta_{11}+\beta_{12}\right) \bar{U}_{, y y}+\left(\beta_{61}+\beta_{62}\right) \bar{U}_{x y} \\
& f_{2}(x, y)=-\left(\beta_{41}+\beta_{42}\right) \bar{U}_{, x}+\left(\beta_{51}+\beta_{52}\right) \bar{U}_{, y}-\frac{A_{43}}{A_{33}} A+\frac{53}{A_{33}} B+2 v .
\end{aligned}
$$

Suivant [4], on peut démontrer que les integrales générales des equations differencielles partielles (7), sont:

$$
\begin{aligned}
& F(x, y)=2 \operatorname{Re}\left[F_{1}\left(z_{1}\right)+F_{2}\left(z_{2}\right)+F_{3}\left(z_{3}\right)\right]+F_{0}(x, y), \\
& \psi(x, y)=2 \operatorname{Re}\left[\lambda_{1} F_{1}^{\prime}\left(z_{1}\right)+\lambda_{2} F_{2}\left(z_{2}\right)+\frac{1}{\lambda_{3}} F_{3}^{\prime}\left(z_{3}\right)\right]+\psi_{0}(x, y)
\end{aligned}
$$


I. T. Mintchev: Probléme de Somigliana sur la théorie de l'élasticité, etc. 187

où $F_{k}\left(z_{k}\right)$ sont les fonctions analytiques des variables généralisées complexes $z_{k}\left(x+\mu_{k} y\right),(k=1,2,3)$, et $\mu_{k}$ sont les racines des equations caractéristiques algebriqne, qui dans ce cas ont la forme suivante:

$$
\begin{gathered}
{\left[\beta_{55} \mu^{2}-\left(\beta_{45}+\beta_{54}\right) \mu+\beta_{44}\right]\left[\beta_{11} \mu^{4}-\left(\beta_{16}+\beta_{61}\right) \mu^{3}+\left(\beta_{12}+\beta_{21}+\beta_{66}\right) \mu^{2}-\right.} \\
\left.-\left(\beta_{26}+\beta_{62}\right) \mu+\beta_{22}\right]-\left[\beta_{15} \mu^{3}-\left(\beta_{14}-\beta_{65}\right) \mu^{2}+\left(\beta_{25}+\beta_{64}\right) \mu-\beta_{24}\right] . \\
\cdot\left[\beta_{51} \mu^{3}-\left(\beta_{41}+\beta_{56}\right) \mu^{2}+\left(\beta_{46}+\beta_{52}\right) \mu-\beta_{42}\right]=0 .
\end{gathered}
$$

On peut facilement démontrer, que dans ce cas les racines des equations caractéristiques, (11) sont toujours complexes ou imaginaires, comme c'est le cas dans la théorie classique de l'élasticité [4].

Quand sont déterminées les fonctions des contraintes $F(x, y)$ et $\psi(x, y)$, d'après les formules (3) et (4) on peut determiner les contraintes et les déformations dans un point quelconque.

\$3. - Puisque le corps étudié a une symetrie élastique qui est normale a l'axe $z$; done $w=$ const et $u=u(x, y), v=v(x, y)$. Pour les composantes des contraintes on obtient:

$$
\begin{aligned}
& \sigma_{x x}=F_{, y y}+\bar{U}, \quad \sigma_{y y}=F_{, x x}+\bar{U}, \quad \sigma_{x y}=-F_{, x y}, \\
& \sigma_{x z}=0, \quad \sigma_{y z}=0, \\
& \sigma_{z z}=-\frac{1}{A_{33}}\left(A_{31} \sigma_{x x}+A_{32} \sigma_{y y}+A_{36} \sigma_{x y}\right) .
\end{aligned}
$$

Donc, ici, on a $\psi=0$ et pour $F(x, y)$ on obtient une équation differencielle partielle à coefficien's variables

$$
\begin{aligned}
\beta_{22} F_{, x x x x} & +\beta_{11} F_{, y y y y}-\left(\beta_{26}+\beta_{62}\right) F_{, x x x y}+\left(\beta_{12}+\beta_{21}+\beta_{66}\right) F_{, x x y y}- \\
& -\left(\beta_{16}+\beta_{61}\right) F_{, x y y}+\left(2 \beta_{22, x}-\beta_{62, y}\right) H_{, x x x}+\left(2 \beta_{11, y}-\beta_{61, x}\right) F_{, y y y}+ \\
& +\left(2 \beta_{12, y}-2 \beta_{26, x}-\beta_{62, x}-\beta_{66, y}\right) F_{, x x y}+\left(2 \beta_{21, x}-2 \beta_{16, y}-\beta_{61, y}+\beta_{66, x}\right) F_{, x y y}+ \\
& +\left(\beta_{12, y y}+\beta_{22, x x}-\beta_{62, x y}\right) F_{, x x}+\left(\beta_{11, y y}+\beta_{21, x x}-\beta_{61, x y}\right) F_{, y y}- \\
& \left.-\left(\beta_{16, y y}+\beta_{26, x x}-\beta_{66, x y}\right) F_{, x y}=-\beta_{21}+\beta_{22}\right) \bar{U}_{, x x}\left(\beta_{11}+\beta_{12}\right) \bar{U}_{, y y}+ \\
& +\left(\beta_{61}+\beta_{62}\right) \bar{U}_{, x y}+\left(\beta_{61, y}+\beta_{62, y}-2 \beta_{21, x}-2 \beta_{22, x}\right) \bar{U}_{, x}+\left(\beta_{61, x}+\right. \\
& \left.+\beta_{62, x}-2 \beta_{11, y}-2 \beta_{12, y}\right) \bar{U}_{, y}+\left(\beta_{61, x y}+\beta_{62, x y}-\beta_{11, y y}-\beta_{12, y y}-\right. \\
& \left.-\beta_{21, x x}-\beta_{22, x x}\right) \bar{U}
\end{aligned}
$$

dans le cas où les forces volumiques ont un potenciel elastique.

Pour la détermination de la fonction des contraintes $F(x, y)$, en pent supposer que les points dans lesquels s'étudie l'état de contraintes et de 
deformation se trouvent sur les lignes isoclines de la section transversale qui peuvent être determinées experimentalement. Dans ce cas l'équation (13) se simplifie et prend la forme:

$$
\begin{aligned}
& \beta_{22} F_{, x x x x}+\beta_{11} F_{, y y y y}-\left(\beta_{26}+\beta_{62}\right) F_{, x x y y}+\left(\beta_{12}+\beta_{21}+\beta_{66}\right) F_{, x x y y}- \\
& \quad-\left(\beta_{16}+\beta_{61}\right) F_{, x y y}=-\left(\beta_{21}+\beta_{22}\right) \bar{U}_{, x x}-\left(\beta_{11}+\beta_{12}\right) \bar{U}_{, y y}+\left(\beta_{61}+\beta_{62}\right) \bar{U}_{, x y} .
\end{aligned}
$$

Ici comme dans la théorie d'elasticité classique on peut montrer que la forme générale de la fonction des contraintes $F(x, y)$ sera

$$
F(x, y)=2 \operatorname{Re}\left[F_{1}\left(z_{1}\right)+F_{2}\left(z_{2}\right)\right]+F_{0}
$$

où $F_{0}$ est une solution partielle de l'équation differencielle partielle non-ho. mogene (14) et $F_{1}, F_{2}$ sont deux fonctions analytiques qui dépendent des racines de l'équation caractéristique

$$
\beta_{11} \mu^{4}-\left(\beta_{16}+\beta_{61}\right) \mu^{3}+\left(\beta_{12}+\beta_{21}+\beta_{66}\right) \mu-\left(\beta_{26}+\beta_{62}\right) \mu+\beta_{22}=0,
$$

qui peuvent être complexes ou imaginaires.

Les expressions des composantes de la contrainte et du deplacement élastique, et les conditions du contonr-definis par la fonction des contraintes $F(x, y)$ restent les mêmes comme dans la théorie de l'élasticité elassique.

On peut étudier analogiquement le problème de l'état de contrainte planécuire. L'éatat de contraiıte planéaire se caractérise suivant la condition $\sigma_{z z}=0$.

L'équation fondementale se détermine par la fonction des contraintes $F(x, y)((13)(14))$, gard sa forme, mais on doit remplacer $\beta_{i j}$ par $A_{i j}$. Par conséquent les expressions des contraintes et des deplacements elastiques restent invariables quand $\beta_{i j}$ est remplacé par $A_{i j}$.

Comme dans la théorie de l'élasticité elassique on peut démputrer, que la solution, du problème de Somighiana pour la théorie d'élasticité dans un milieu a modules différents, existe et est unique.

\section{LITTERATURE}

[1] C. Somigliana, Ricerche sulla deformazione ed $i$ fenomeni piezoelettrici in uno cilindro cristallino, Annali di Matem. pura ed appl., IT, t. XX, 1892, Milano.

[2] W. VoIgт, Lehrbuch der Kristallphysik, Leipzig-Berlin (Teubner), 1928.

[3] S. G. LECHArckr, Nekotorie sloutchai ouprongogo rovnovesia ednorodnogo cilindra s proi. zvolnoy anisotropiey, Prikladniaia matematika, t. II, vip. 3, 1939, SsSR.

[4] - -, Teoria ouprogosti anisotropnogo tela, Gostechizdat M-L. 1950, SSSR.

[5] I. T. MINTohev, Osnouni ouravnenia za rasnomodoulnata teoria na elestitchnosta $z \alpha$ ortogonalna.anisotropna sreda, Godichnik na VTUZ Prilogna mechanika, 1968. 